\title{
Transmission electron microscopic identification of silicon-containing particles in synovial fluid: potential confusion with calcium pyrophosphate dihydrate and apatite crystals
}

\author{
THOMAS BARDIN ${ }^{1}$ H. RALPH SCHUMACHER,${ }^{2}$ JULIETTE LANSAMAN, \\ SUSAN ROTHFUSS, ${ }^{2}$ AND ANTOINE DRYLL ${ }^{1}$ \\ From the ${ }^{1}$ Clinique Rhumatologique de l'Hôpital Lariboisière, Paris, and the ${ }^{2}$ Veterans Administration \\ Medical Center and University of Pennsylvania, Philadelphia, USA
}

SUMMARY Silicon-containing particles were identified by transmission electron microscopy (TEM) in thin sections of two synovial fluids, which also contained calcium pyrophosphate dihydrate (CPPD) crystals, aspirated during acute attacks of pseudogout. Such particles, which are interpreted as probably being artefacts from glassware, were electron dense and similar in appearance to some CPPD or hydroxyapatite crystals.

Transmission electron microscopy (TEM) is, when available, a useful technique for studying crystal related arthropathies. It can show crystals too small to be seen by light microscopy ${ }^{1-4}$ and allows further study of crystal-cell interaction. However, it can also display various artefacts, which may be confusing. ${ }^{5}$ We report here the TEM observation of silicon containing particles which were found in synovial fluid preparations. These particles might have been confused with calcium pyrophosphate dihydrate (CPPD) or other crystals. We believe they were artefacts from glassware.

\section{Materials and methods}

Synovial fluids from two patients were studied. They both contained crystals suggestive of CPPD by compensated polarising light microscopy and were processed for TEM as part of a study of CPPD deposition disease. No other unidentified particles were noted on initial examination of these fluids as wet preparations.

Patient 1 was an 83-year-old woman who had had several episodes of acute arthritis of her knees. Chondrocalcinosis of the knees, ankles, and shoulders was seen on $x$-rays. The fluid included in this report was aspirated during an acute knee arthritis.

Accepted for publication 23 February 1984.

Correspondence to T. Bardin, MD, Clinique Rhumatologique de l'Hôpital Lariboisière, 6 rue Guy Patin, 75010 Paris, France.
It contained $20.8 \times 10^{9}$ white cells/l. Patient 2 was a 76-year-old female who had repeated attacks of pseudogout of her left ankle without any radiological chondrocalcinosis. Synovial fluid included in this report was aspirated during one of these attacks. It contained only $0.02 \times 10^{9}$ white cells $/$. Neither of the patients had any underlying disease known to be associated with CPPD deposition. Associated diseases included senile dementia in case 1 and renal phosphate wasting with normal serum parathormone (PTH) level in case 2.

The two synovial fluids were aspirated with disposable plastic syringes without the use of sterile gloves, then collected in glass vials containing glass balls which were mixed with the fluids to prevent coagulation before standard analysis. For electron microscopy the fluids were then transferred to glass tubes and centrifuged. Pellets were quickly placed in $5 \%$ glutaraldehyde for half an hour at $4^{\circ} \mathrm{C}$, then minced in $1 \times 1 \mathrm{~mm}$ pieces. Pieces were fixed in $2.5 \%$ glutaraldehyde at $4^{\circ} \mathrm{C}$ for two hours, washed in cacodylate buffer, postfixed in $2 \%$ osmium tetroxide, dehydrated in graded series of alcohol, and embedded in Spurr medium. Thin sections were cut with a diamond knife and observed in a Zeiss EM 20 transmission electron microscope. $X$-ray dispersive microanalysis was performed on unstained carbon-coated thin sections in the same TEM operated at $60 \mathrm{kV}$, with an $\mathrm{Si}$ (Li) $x$-ray detector interfaced with a multichannel analyser and a computer system. 
Borosilicate and limeglass pipette fragments were ground and also embedded in Spurr medium. Thin sections of these materials were studied by TEM in a similar way to the two synovial fluids.

\section{Results}

CPPD crystals were seen in both synovial fluids. By TEM they appeared as electron dense rectangles of

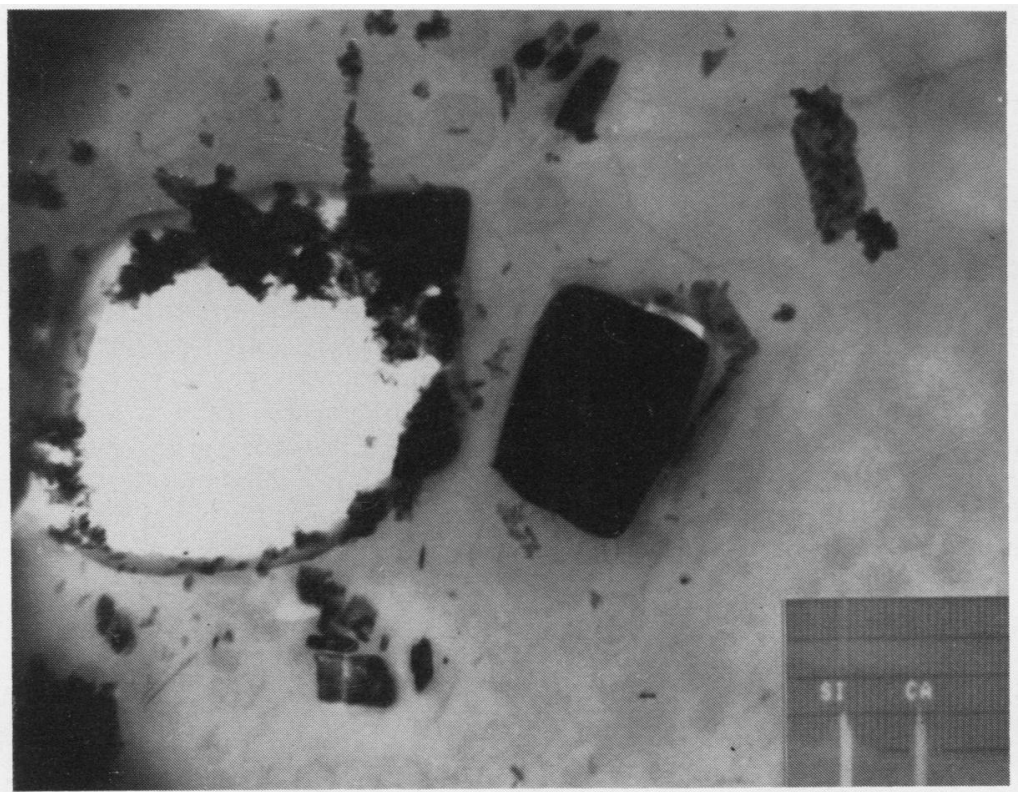

Fig. 1 Dense quadrangular silicon-containing particle which is located either inside the cell or in an invagination. (Unstained thin section, $\times 16270) . X$-ray microanalysis showed this particle to contain silicon and calcium (insert).

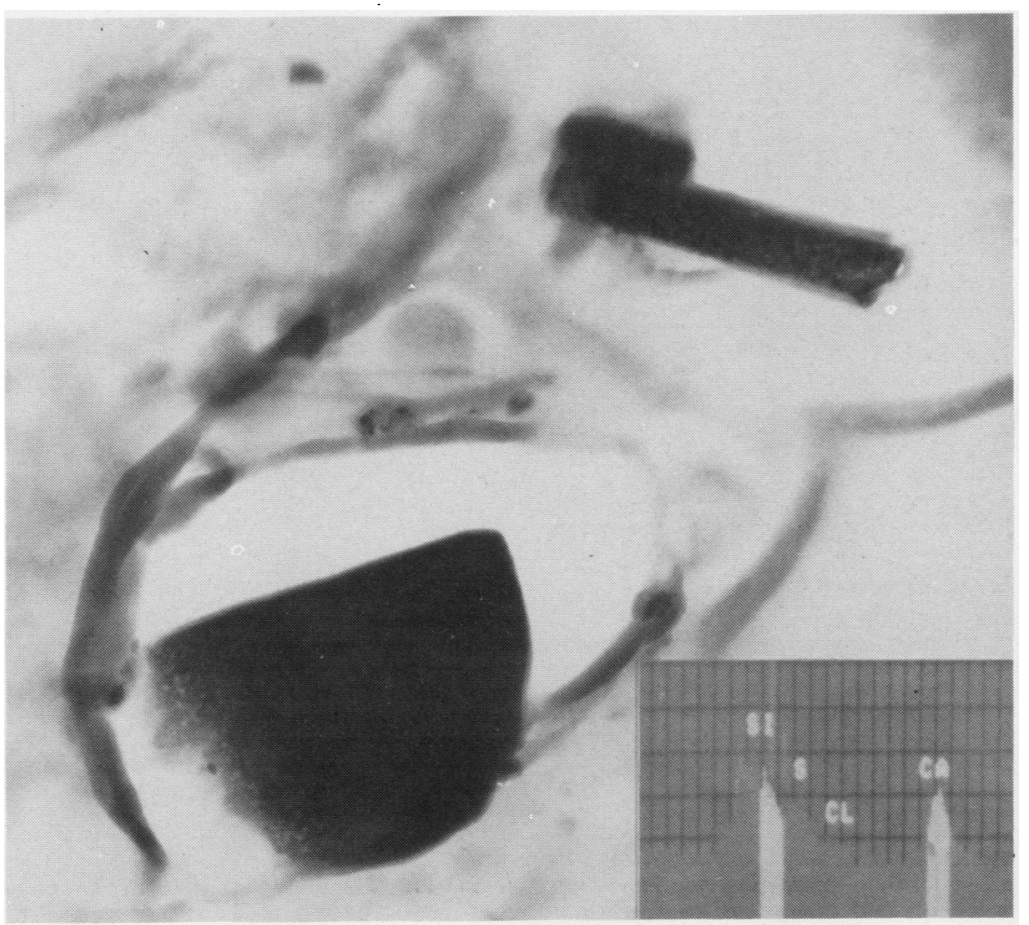

Fig. 2 Dense siliconcontaining particles misleadingly similar to $C P P D$ crystals. $(\times 11$ 110). These particles also contained calcium, chlorine, and sulphur (insert). 
varying length, which became foamy during exposure to the electron beam. $X$-ray microanalysis showed that they contained calcium and phosphorus with a $\mathrm{Ca} / \mathrm{Pratio}$ approximating to one. Other electron dense particles were found to contain silicon (Fig. 1). Their morphology was often very suggestive of CPPD crystals except that they did not become increasingly foamy under the electron beam. Thin sections of some silicon-containing particles did reveal a fine pitting with uniform holes that did not change with beam exposure (Fig. 2). Siliconcontaining particles could also appear as more fragmented masses sometimes divided into small spicules (Fig. 3). Some such spicules could also be potentially confused with apatite. $X$-ray microanalysis showed that these particles often contained small amounts of calcium, and more rarely of sulphur and chlorine, in addition to silicon. CPPD crystals were both intra- and extracellular. Silicon-containing particles were generally not found inside cells. One silicon-containing fragment possibly related to a cell was seen in one unstained section (Fig. 1).

TEM studies of the pipette fragments showed that the glass debris obtained were electron dense and contained silicon. Varying amounts of calcium in addition to silicon were found in limeglass pipette samples.

\section{Discussion}

This study has identified silicon-containing particles in thin sections obtained from two synovial fluids. The morphology of these particles was diverse. Before microanalysis some of them were mistaken for crystals and in particular for dense CPPD crystals. However, the silicon-containing particles did not become foamy during the electron beam exposure as did CPPD crystals. An attentive observer could thus differentiate the two materials, though CPPD crystals are not the only crystal type to have such a similar dense ultrastructure. Oxalate crystals are known to have a close TEM resemblance to $\mathrm{CPPD},{ }^{6}$ so that microprobe analysis or other analytical techniques ${ }^{7}$ are required for definite crystal identification.

The finding of silicon in synovial fluids raises the question of its origin and possible pathogenic role. Silica particles can absorb proteins ${ }^{8}$ and are known to interact with macrophages. They can kill by a 'perforation from within' mechanism ${ }^{9}{ }^{10}$ and are believed to cause pulmonary lesions in silicosis. However, silica particles have never been held responsible for any otherwise unexplained arthritis. In our patients we have no evidence that the silicon-containing particles are responsible for their joint inflammation. In both cases CPPD crystals

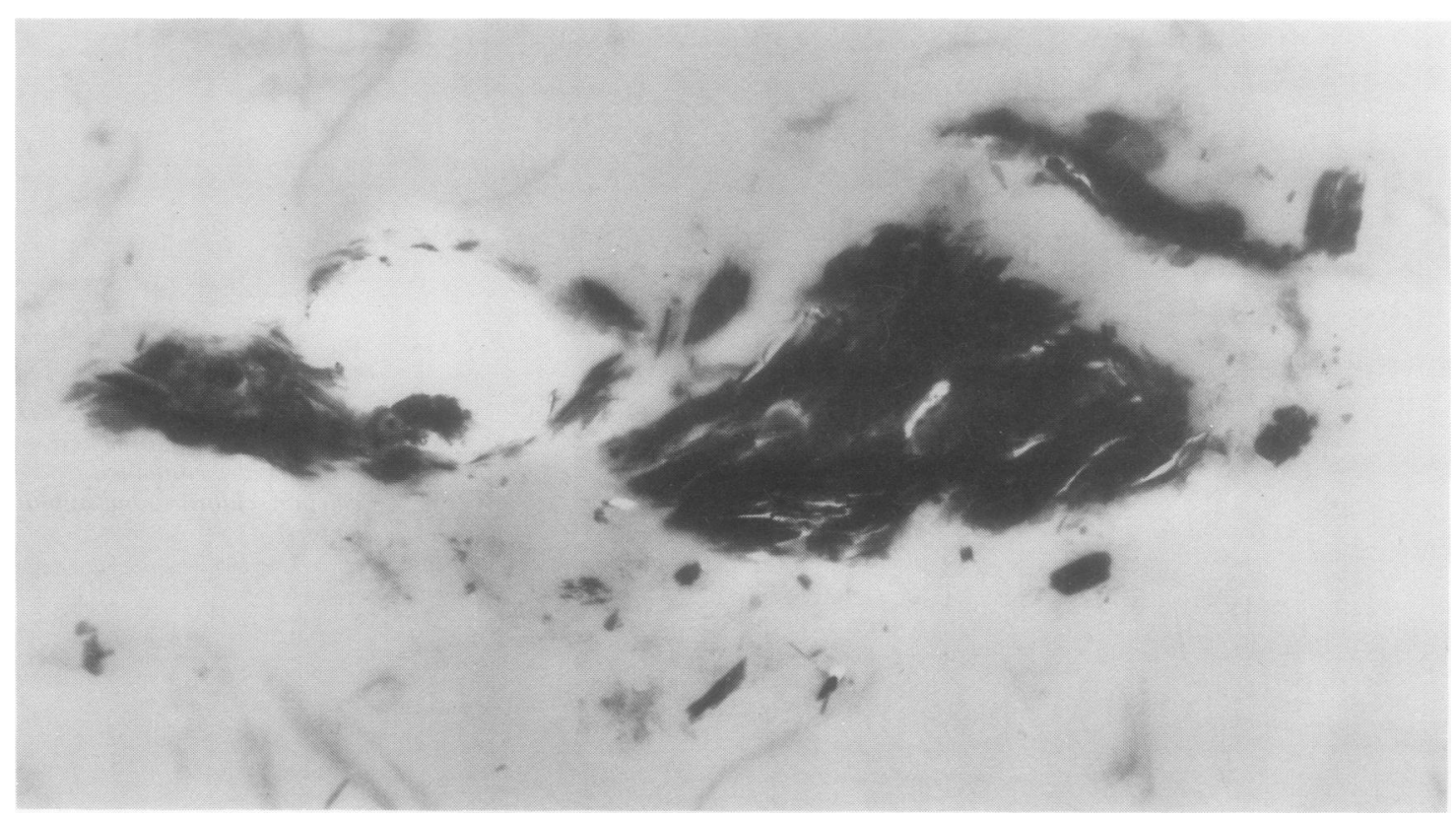

Fig. 3 Silicon-containing particles fragmented into spicules which can be potentially confused with apatite crystals. These particles did not contain any detectable element other than silicon. $(\times 6750)$. 
were also present and could account for the acute arthritis. In one fluid a silicon-containing particle was possibly within a cell, but phagocytosis can occur in vitro as well as in vivo. Silica-containing particles can break off silicone joint prostheses and appear in joint tissues, ${ }^{11}$ but in neither of our patients was the presence of silicon in a joint easily explainable. Neither patient had a history of any obvious source of silica exposure or intake. ${ }^{12}$ On the other hand several steps in the processing of the synovial fluid could explain the presence of silicon in the final preparation. Disposable syringes were used to aspirate synovial fluids. They contain a silicon compound (polymethylsiloxane), serving as a lubricant,${ }^{13}$ which could account for minute amounts of silicon in the fluids. Moreover, synovial fluids were collected on glass balls to prevent clotting, and glassware was used during the preparation for TEM. Pipettes containing limeglass were used at several steps of the processing and could account for the frequent association of calcium with the siliconcontaining particles. Therefore it seems highly probable that the variously shaped silicon-containing particles were artefacts from glassware. Investigators should be aware that such artefacts can have an appearance on transmission electron microscopy that can mislead one into suspecting CPPD or apatite crystals.

\section{References}

1 Schumacher H R, Jimenez S A, Gibson T, et al. Acute gouty arthritis without crystals identified on initial examination of synovial fluid. Report on nine patients. Arthritis Rheum 1975; 18: $603-12$.

2 Honig S. Gorevic P. Hoffstein S. Weissmann G. Crystal deposition disease. Diagnosis by electron microscopy. Am J Med 1977; 63: 161-4.

3 Dieppe P A. Huskisson E C. Crocker P, Willoughby D A. Apatite deposition disease. A new arthropathy. Lancet 1976; i: 266-8.

4 Schumacher H R, Somlyo A P. Tse R L, Maurer K. Arthritis associated with apatite crystals. Ann Intern Med 1976; 87: 411-6.

5 Schumacher H R. Pathology of the synovial membrane in gout. Light and electron microscopic studies. Interpretation of crystals in electron micrographs. Arthritis Rheum 1982: 18: 771-82.

6 Hoffman G S. Schumacher H R. Paul H. et al. Calcium oxalate microcrystalline associated arthritis in end stage renal disease. Ann Intern Med 1982; 97: 36-42.

7 Dieppe P. Calvert P. Crystals and joint disease. London: Chapman and Hall, 1983: 57-88.

8 Scheel L D. Smith B. Van Riper J. Fleisher E. Toxicity of silica. II Characteristics of protcin films absorbed by quartz. Arch Industr Hyg Occup Med 1953; 9: 29-36.

9 Allison A C. Lysosomes and the toxicity of particulate pollutants. Arch Intern Med 1971: 128: 131-9.

10 Nadier S, Goldfisher S. The intracellular release of lysosomal contents in macrophages that have ingested silica. $J$ Histochem Cytochem 1971: 18: 368-71.

11 Rosenthal D 1. Rosenberg A E. Schiller A L. Smith R J. Destructive arthritis due to silicone: a foreign-body reaction. Radiology 1983: 149: 69-72.

12 Levison D A, Banim S. Crocker P R. Wallace D M A. Silica stones in the urinary bladder. Lancet 1982; i: 704-5

13 Wilckham M G. Rudolph R. Abraham J. Silicon identification in prothesis associated fibrous capsules. Science 1978: 199: $437-9$. 